\title{
Feature Point Selection using Structural Graph Matching for MLS based Image Registration
}

\author{
Hema P Menon \\ Department of CSE \\ Amrita Vishwa Vidyapeetham \\ Coimbatore \\ Tamil Nadu - 641 112, India
}

\author{
K A Narayanankutty \\ Department of ECE \\ Amrita Vishwa Vidyapeetham \\ Coimbatore \\ Tamil Nadu - 641112, India
}

\author{
Indulekha T S \\ Department of CSE \\ Amrita Vishwa Vidyapeetham \\ Coimbatore \\ Tamil Nadu - 641112, India
}

\begin{abstract}
Image registration is a method of determining a mapping or a transformation that relates positions in one image, to the corresponding positions in the other images under considerations. The process of registration depends on the homologous control points that are selected from the source and the target images. This paper focuses on the use of the structural information of an image, for selecting control points, as it remains the same even when it undergoes most of the transformation and illumination changes. The points thus obtained are then given to the Moving Least Squares (MLS) based registration technique reported earlier by the authors [11].
\end{abstract}

\section{Keywords}

Image Registration, Moving Least Squares, Graph Marching, Delaunay Triangulation, Hungarian Method

\section{INTRODUCTION}

Feature detection and feature matching plays an inevitable role in the process of image registration. The accuracy of any registration scheme completely relay on the feature point detection and feature matching method. The better the feature detection the better the matching will be and a good feature matching leads to an efficient image registration.

In literature there are many methods available for feature detection and feature matching. But exploiting the structural information of images for feature detection and matching are not common. Structural information of an image is important as it remains the same even if the image undergoes most of the transformations like rotation, scaling etc. So if a method uses the structural information for matching, it can yield good result in almost every condition and thereby necessitating a need for structural representation of images.

Graphs as a data structure can very well represent the structure of an image. If a graph is translated, rotated or transformed it is still the same graph in the mathematical sense. These invariance properties of graphs make them attractive for various applications. Graphs are invariant for most of the transformations. And this invariance property makes them attractive for many applications.

As image registration is concerned about the spatial or geometrical alignment of the images, the first step in any image registration mechanism is the feature point extraction and feature point matching. And since the performance of any image registration method is completely dependent on feature matching, it is very essential that the features are matched as properly as possible. This leads to the need for a feature matching method that can guarantee the accuracy.

The proposed methodology uses structural information of the target and source images for feature matching. For exploiting the structure, images are represented as graphs. Among the available graph structures, Delaunay triangulations and adjacency matrices are used for the graph construction of images. Each vertex of the Delaunay are considered to be the nodes of the graphs. Now the feature matching problem turns into a graph matching problem.

In this paper, a novel graph matching algorithm Vertex Degree Neighborhood (VDN) algorithm has been proposed for image registration. In this method a first level matching is done using the degree and neighborhood structure of each node for finding the initial match. This is then improved/ optimized by a second level feature matching, by considering a feature vector which includes the features like entropy, mean and standard deviation of the neighborhood region of each node.

\section{LITERATURE SURVEY}

Image registration is a process of determining the correspondence of features between images collected at different times or using different imaging modalities. The correspondences can be used to change the appearance by rotating, translating, stretching etc of one image so that it more closely resembles another and so the pair can be directly compared, combined or analyzed. As image registration is concerned about the spatial or geometrical alignment of the images, the first step in any image registration mechanism is the feature point extraction and feature point matching. Since the performance of any image registration method is completely dependent on feature matching it is very essential that the features are matched as properly as possible.

Image registration can be broadly classified into three categories, namely intensity based image registration, feature based image registration and structural image registration. Many research works are available in the field of Intensity and feature based image registration. A feature based retinal image registration framework was proposed by Chen, Jian et,al in [1]. It detects the features like vascular bifurcations using the Partial Intensity Invariant Feature Descriptor (PIIFD) framework and then registration is performed by Harris PIIFD. It is claimed in this paper that the acceptable registration obtained is $89.9 \%$ and the effective registration is $99.4 \%$. Jian Zheng et.al proposed a new method for retinal image registration using salient feature region computation method [2]. Here the similarity measure used for registration is normalized cross correlation. The average registration 
accuracy obtained is 2.50 pixels with standard deviation of 2.17 pixels. An Edge Driven Dual -Bootstrap Iterative Closest Point Algorithm was proposed by Tsai et al. for registration[3]. In this algorithm it first computes the bootstrap region over the image and in each bootstrap region it iteratively refines the transformation estimates and expands the bootstrap region. Here the average accuracy obtained is claimed to be 2.05 pixels and standard variation is 1.57 pixels.

Recent developments are exploiting the structure of the Images for the registration purpose. Structure based image registration overcomes most of the disadvantage of intensity and feature based methods. One such method for the structural representation of an image is Laplacian eigenmaps and has been discussed by Christain et al [4]. Structural representations, allows the direct application of L1 and L2 norms. In this method, Laplacian eigenmaps search for similar patches in high-dimensional patch space and embed the manifold in a low-dimensional space under preservation of locality. This can be interpreted as the identification of internal similarities in images. Internal similarity across images is comparable and yields a very good registration results for the new structural representation.

A popular graph based registration approach deals with the concept of graph- cuts for registration. Tang and Chung modeled the non rigid image registration problem as a graph cut based energy minimization framework [5]. The optimization was done by using the graph cut algorithm via $\alpha$ expansions. The dissimilarity measure used in the energy function of this graph cut based method was restricted to the sum of absolute difference (SAD) and the sum of squared distances(SSD).

The energy minimization framework proposed Ronald [6], uses the mutual information as the similarity measure. The method was an approximation of MI to the graph-cuts based method. Since mutual information can capture complex statistical relationships between the intensities of the image pair without a priori knowledge of those relationships exploiting the mutual information is valuable.

Another area of graph matching uses graph concepts such as graph isomorphism, sub-graph isomorphism and maximum common sub-graph for finding the matching. Graph isomorphism can be used to find out if two objects are the same. Sub graph isomorphism can be used to find out if one object is part of another object or if one object is present in a group of objects.

The standard algorithm for graph and sub-graph isomorphism detection is the one, where maximum common sub-graph detection has been addressed. These methods use the concepts of scene graph and model graph or can be called as source graph and target graph. The purpose of this algorithm is to find the mapping from the source graph to the target graph.

Classical error correcting sub-graph isomorphism methods discussed by Tsai[7] and Sanifeliu[8] use particular versions of $\mathrm{A}^{*}$ search procedure to find the best matching. These methods are guaranteed to find the optimal matching, but require exponential time due to NP-completeness of the problem. The inexact graph matching procedure proposed by Hlaoni[9] decomposes the matching process into $\mathrm{K}$ phases, where the value of $K$ ranges from 1 to the minimum of the numbers of nodes in the two graphs to be matched. The efficiency of this algorithm results from the use of small values of $\mathrm{K}$ significantly reducing the search space while still producing very good matching between graphs.
Schelewald[10] has used the semi definite programming for sub graph matching. Shi.et.al [12] proposed an objective function in terms of normalized cut (Ncut) where the cut cost is computed as a fraction of the total edge connections to all nodes in the graph. Here grouping is done by minimizing the disassociation between the groups and maximize the association within the groups for segmentation purpose. The same criteria can be extended for feature based image registration also.

The disadvantages of both the intensity and feature based methods can be reduced in structure based image registration. Since the structural information of a patch or an image is only dependent on the structures in the patch not the intensity values with which the structure is displayed. Structural representation is invariant to many transformations and even for multimodal images the structural representation remains the same.

\section{GRAPH BASED FEATURE MATCHING}

In general graphs are considered to be as the powerful data structure for the representation of objects and concepts. They exploit the structural features of objects they represent. In a graph representation the nodes typically represent objects or parts of objects, while the edges describe relation between objects or object parts. The invariance properties of graphs make them a powerful data structure. The interesting invariance properties of graphs are that if they are translated, rotated or transformed into its mirror image it is still the same graph in the mathematical sense. The invariance properties as well as the fact that graphs are well suited to model objects in terms of parts and their relations make them very attractive for various applications.

When graphs are used for representation of structured objects then the problem of measuring object similarity turns into the problem of computing the similarity of graphs, which is also known as graph matching. Now the image registration problem becomes a graph matching problem. Hence graph construction becomes the basic step for performing registration. This paper proposes few methods for graph construction and graph matching.

In mathematical sense graph is a representation of nodes and edges. Graphs can be constructed in many ways. In a graph representation the nodes typically stands for objects or parts of objects. So the first step in graph construction is the identification of object parts or feature points from the image. The interesting points on the objects can be extracted to provide a feature description of the object in the image. Likewise by extracting important feature points and by constructing a graph the structure of an image can represented. This can be done using many feature point detection methods. Some of the commonly used methods are Harris, SURF and SIFT algorithms.

\subsection{Feature Detection Scheme}

The local features in an image can be identified using many different methods. Among them three most commonly used feature detectors are-Harris point detector, SIFT detector, SURF detector.

Scale invariant feature transform is an algorithm to detect and describe local features in images. The algorithm follows Lowe's method for image feature generation. This method transforms an image into a large collection of feature vectors, each of which is invariant to image translation, scaling, and 
rotation, partially invariant to illumination changes and robust to local geometric distortion. Key locations are then located or defined as maxima and minima of the result of the Gaussian function applied in scale space. Low contrast candidate points and edge response points along an edge are discarded. Dominant orientations are assigned to localized key points.

SURF is a local feature detector; it uses an integer approximation to the determinant of Hessian blob detector. It is computed quickly with an integral image. For features, it uses the sum of the Haar wavelet response around the point of interest.

Harris corner points are derived from auto-correlation function with the Taylor expansion and eigenvalue method. The basic idea of this detector is to use the auto-correlation function in order to determine locations where the signal changes in two directions. It is strongly invariant to rotation, scale, illumination variation and image noise.

Among these feature detectors, Harris feature detector is chosen for feature point extraction and graph construction as its invariance properties suit for the image registration application.

\subsection{Graph Construction}

Image Graphs can be constructed in many ways. Mathematically graphs can be represented by adjacency list, adjacency matrix, incidence matrix, Laplacian Eigenmaps, Delaunay triangulation, minimum spanning trees, Voronoi Diagram, trees etc. The choice of graphs depends on the requirement of application. In this work the adjacency matrix and Delaunay triangulation are considered as the graph structure for image representation.

\subsubsection{Adjacency Matrix}

An adjacency matrix is a means of representing which vertices or nodes of a graph are adjacent to which other vertices. In the adjacency matrix A of a graph $G$, the nondiagonal entry $a_{i j}$ is the number of edges from vertex $i$ to vertex $j$, and the diagonal entry $a_{i i}$, is either once or twice the number of edges (loops) from vertex $i$ to itself.

\subsubsection{Delaunay Triangulation}

For a points set $P$ in the 2D Euclidean space, a Delaunay triangulation in the plane is a triangulation $D T(P)$, such that no point in $P$ is inside the circumcircle [21] of any triangle in $D T(P)$. Delaunay triangulations maximize the minimum angle of all the angles of the triangles in the triangulation. Thus avoids having triangles that are very long and thin. The triangular mesh has the advantage of minimizing the distance over which interpolations must take place. This property guarantees that the shape of the Delaunay triangles is the best possible for that set of points. Here the standard incremental construction algorithm is used for constructing Delaunay triangulation.

\subsection{Graph Matching}

After feature point extraction and graph construction now the image registration problem becomes a graph matching problem. In this paper a novel method exploiting the degree and neighborhood structure of each node is used for matching the points in the images to be registered. This method has been named as Vertex -Degree- Neighborhood method for graph matching by the authors, which is then used for control point selection for image registration using Moving Least Squares (MLS).

\subsubsection{Vertex-Degree-Neighborhood based graph matching.}

This method follows a feature based approach for graph matching. It is a two level feature matching method. The method starts by identifying the feature points in the images using Harris detector which is followed by graph construction. The Delaunay triangulations are used for graph construction.

The method consists of two levels. In the first level - the features considered are degree and neighborhood structure of each vertex. An initial set of matching are obtained in this level. In the second level these initial set of matching are improved by using the features-entropy, mean and standard deviation. Here the neighborhood region's properties have been considered for matching. In this second level the optimal graph matching is obtained. Now these optimal matching can be fed into any registration mechanism.

\section{IMPLEMENTATION}

\subsection{Dataset for Graph Matching and} Registration

The experiments were conducted on pairs of brain MRI images. All the images are converted from DICOM to JPEG and TIFF. Images are of size $256 \times 256$.
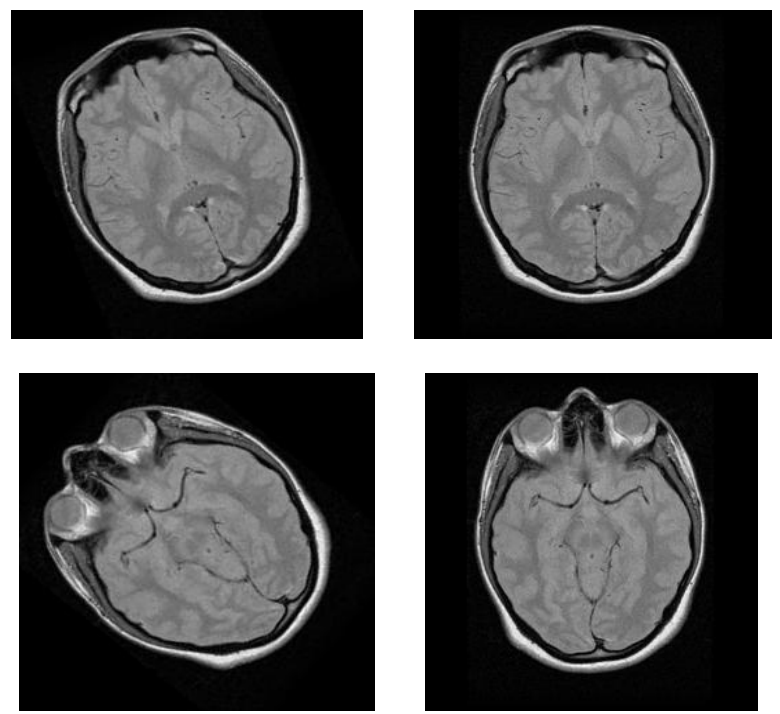

Figure 1: Dataset for image registration

\subsection{System Design}

The system design for then structural based registration is given in figure 2 . The control points selected from the source and target images, using the V-D-N method are given to MLS registration to obtain the registered source image.

\subsubsection{System Design}




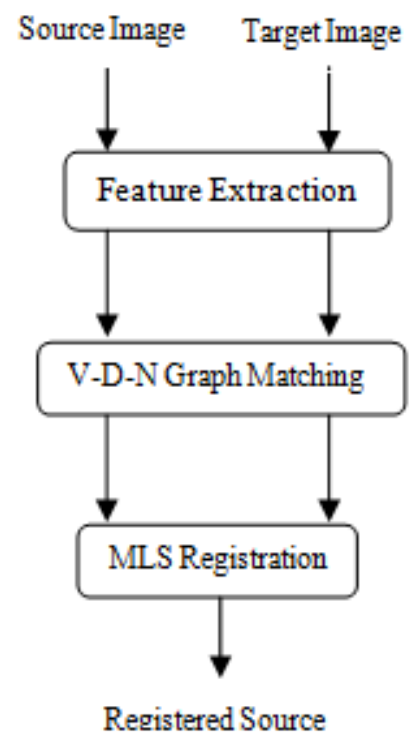

Figure 2. Overall System Diagram

\subsection{Graph Matching by Vertex-Degree- Neighborhood Method}

The first step in the VDN method is the feature point detection. The salient feature points in both the source and target images are identified using the Harris point detection method. With these detected points as the nodes, a Delaunay triangulation is created for both the source and the target images. Now the graph matching problem becomes the node mapping problem of these source and target Delaunay's. This is done in a two level feature matching scheme.

\section{Level 1:}

In the first level a degree based matching of nodes is performed. Using Delaunay triangulation the weighted adjacency matrices for two images is created and also the edge details associated with each vertex is recorded. The weight is assigned by using the Euclidian distance transform norm. In the next step, the degree of each vertex is computed and the edge details associated with it is recorded. Also the neighborhood structure [degree] details of each and every node are stored. Now the first level matching is done by checking the degree of every individual node and of its neighbors.

A vertex ' $P$ ' in the source Delaunay is considered to be mapped to the ' $Q$ ' vertex in the target Delaunay when the degree of $\mathrm{P}$ and its neighbors is same as that of $\mathrm{Q}$ in the target. It is important that, not only the individual vertex degree but also their neighborhood structure need to be matched i.e.; it is a must that the degree of the vertex and its neighbors are same. This ensures that no two non identical nodes are mapped. In this way the first level matching is obtained. But there arise a possibility that there are more than one vertex with which the structure of $\mathrm{P}$ matches in the target, which means that $\mathrm{P}$ can be assigned to more than one node in $\mathrm{Q}$. These multiple matches have to be avoided as a one-to-one correspondence is needed for perfect registration. Hence a second level matching process has been carried out based on the statistical properties.

\section{Level 2:}

In the second level, the initial set of matching's obtained from the first level are only taken into concern, all the other mismatching are avoided at the first level itself. This evidently decreases the number of points under consideration. This level is concerned with a feature based matching using the features: entropy, mean and standard deviation. For that a region based approach is done. Around each point a region is considered by introducing the window concept and compute the above mentioned feature i.e., entropy, mean and standard deviation Then for the vertex $\mathrm{P}$ the entire candidate point matching's are taken and the matching vertex neighborhood region having the properties like P's neighborhood region is selected using a threshold value. Then that vertex is declared as P's optimal matching. Likewise the matching for every other vertices are computed. Now this optimal set of matching can be feed into any registration scheme as required for the image registration application under concern. In this work the MLS based registration method has been used for registering the source and the target images. The system flow diagram for the V-D$\mathrm{N}$ method is shown in Figure 3.

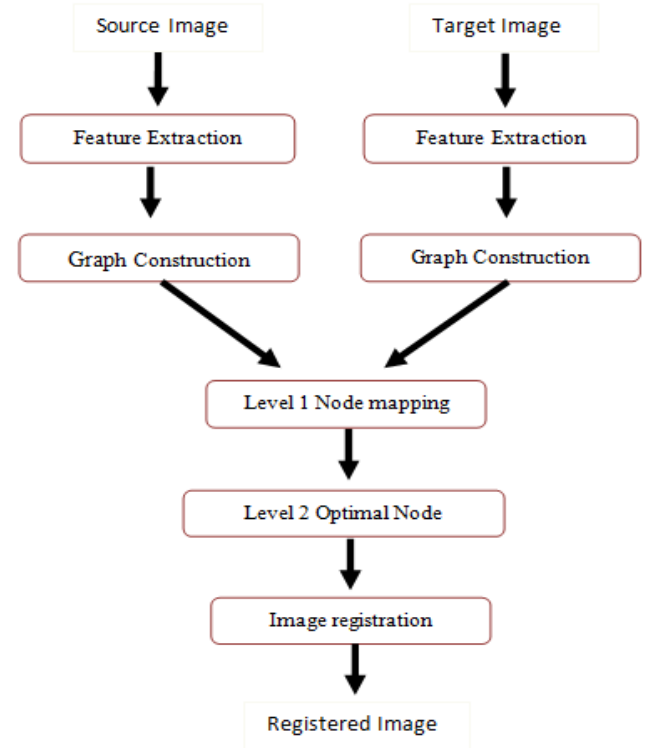

Figure 3. System flow diagram of V-D-N method

\subsection{Evaluation Parameters}

The parameter used for evaluation of the registration process is the Target Registration Error (TRE).

Target Registration Error (TRE) is taken as the evaluation parameter for image registration by graph matching. TRE is defined [Wikipedia] as the displacement between two corresponding points after registration.TRE is calculated at some point of interest mostly at some anatomical positions. A lower TRE indicates a better method. In this case TRE is used to measure how good the points are matched for performing image registration. It is normalized between $[0,1]$.

\section{EXPERIMENTAL RESULTS AND ANALYSIS 5.1 Feature Detection 5.2.1 Feature Point Detection using Harris}



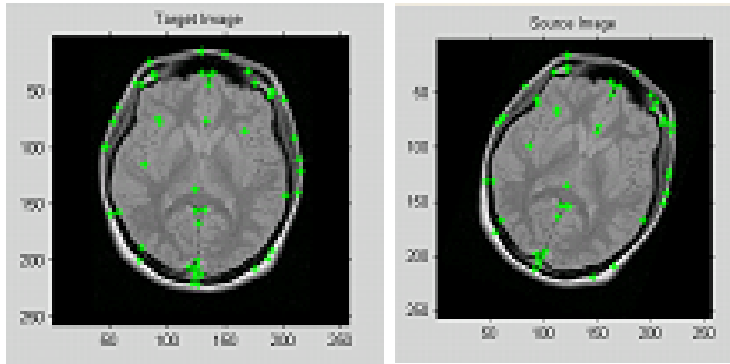

Figure 4 (a): Feature extraction by Harris on sample data set 1.
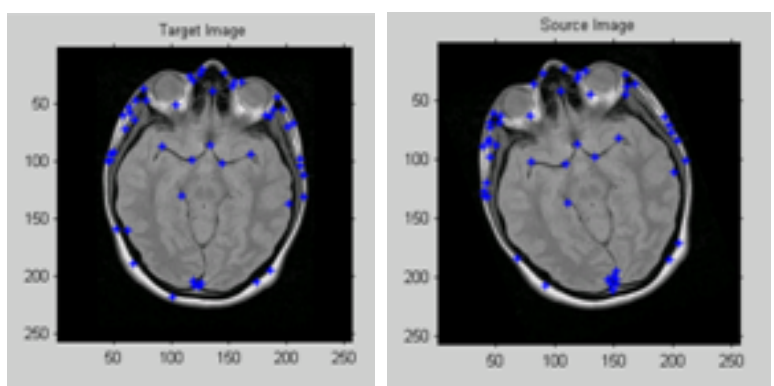

Figure 4(b): Feature extraction by Harris on sample data set 2 .

\subsubsection{Graph Construction}
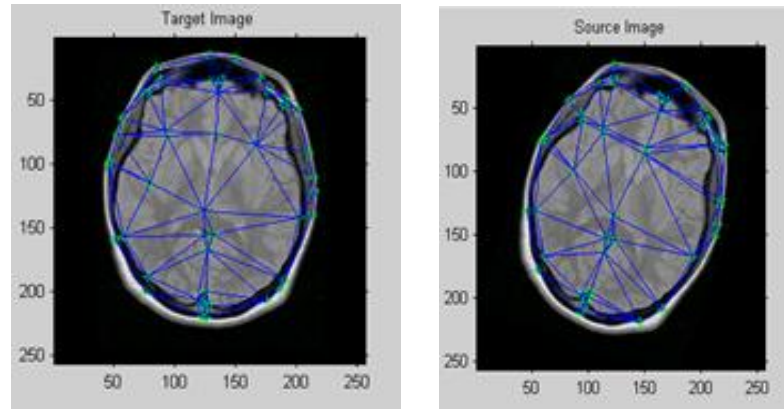

Figure 5(a): Delaunay Construction on sample data set 1.
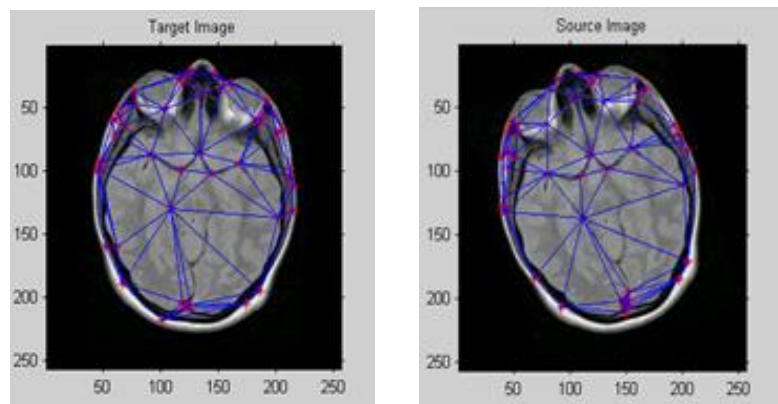

Figure 5(b): Delaunay Construction on sample data set 2.

\subsubsection{Graph Matching By $V-D-N$}

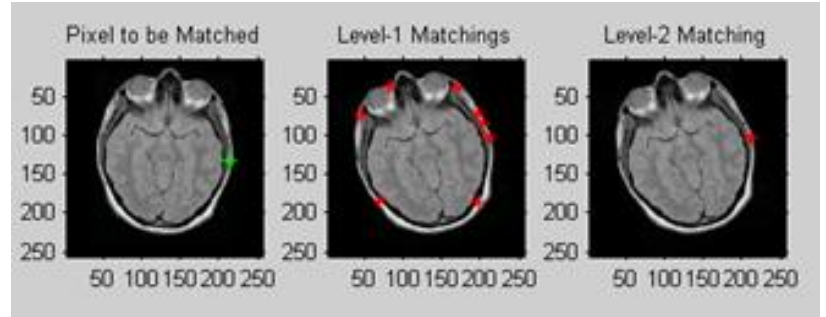

Figure 6 (a): Node by Node Matching by V-D-N Method on sample data set 1 .

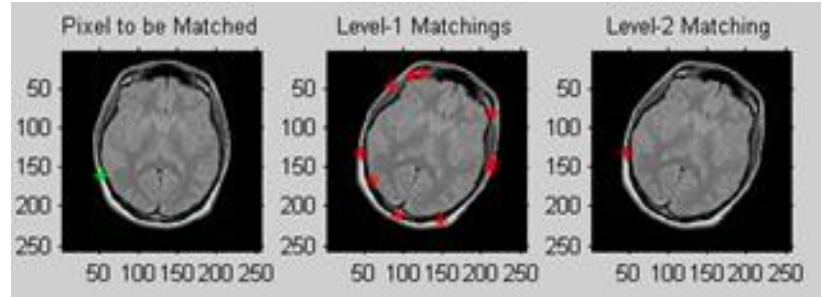

Figure 6 (b): Node by Node Matching by V-D-N Method on sample data set 2 .

\subsubsection{Image registration using $M L S$}

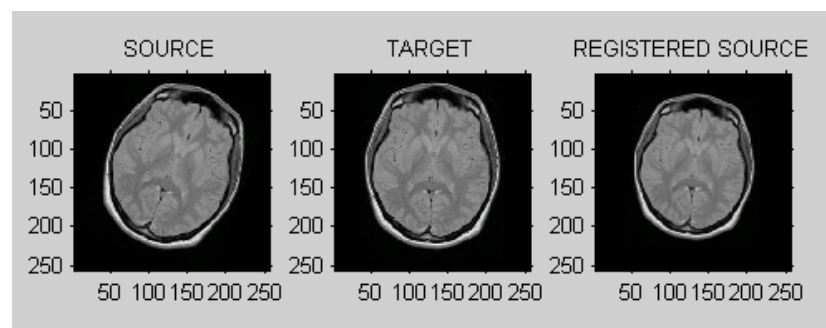

Figure 7(a): Registration using MLS for sample data set 1.

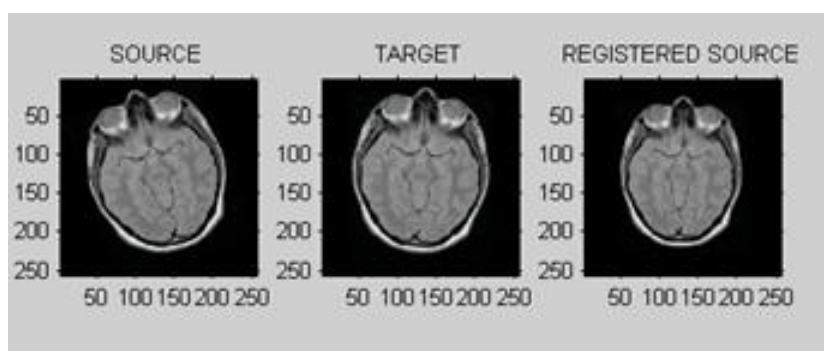

Figure 7(b): Registration using MLS for sample data set 2.

\subsubsection{Target registration Error}

The VDN method is best suited for images that are smaller in size as the processing is done node by node. The registration accuracy achieved is almost as that of the Hungarian method. In some cases, when the brain image considered had lot of details in it, for example like the mid axial or sagittal MRI slice, the accuracy of the proposed method drops, because of the intrinsic features present in the images. Figure 8 gives a comparison chart of the TRE values obtained for representative set of test images. 


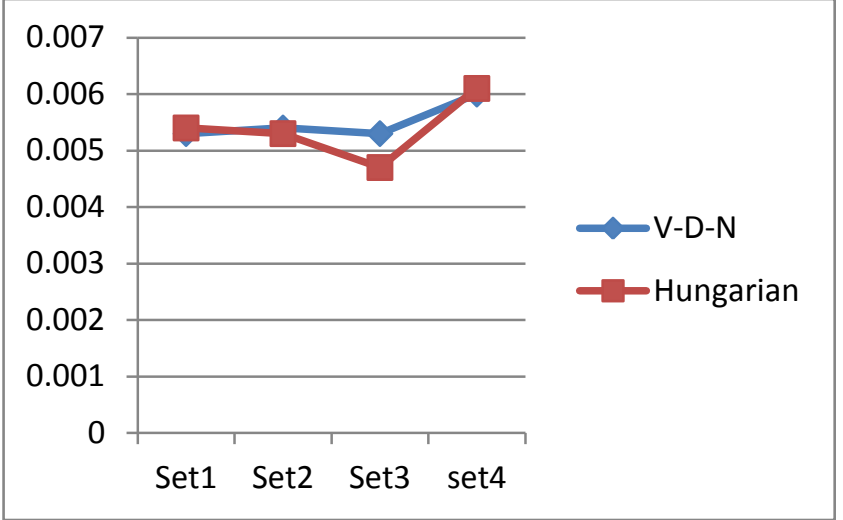

Figure 8: TRE Evaluation of V-D-N and Hungarian Graph Matching

\section{CONCLUSION}

This work proposes a graph matching approach namely, Vertex-Degree-Neighborhood (VDN) method which uses the structural information in the image for the image registration. The initial control points were obtained using Harris corner detector. In this work the Delaunay triangulation method was used for creating the graph structure. The correspondence points between the source and target images were selected by the VDN approach. The points thus obtained were used as the control points for MLS based image registration technique. The performance assessment of the method was done by computing the TRE between the target image and the registered source image. The results are compared with the Hungarian method. The efficiency of the control point selection and matching can further be improved by optimizing using Semi Definite Programming.

\section{REFERENCES}

[1] Chen, Jian, et al. "A partial intensity invariant feature descriptor for multimodal retinal image registration." Biomedical Engineering, IEEE Transactions on 57.7 (2010): 1707-1718.

[2] Zheng, Jian, et al. "Salient feature region: a new method for retinal image registration." Information Technology in Biomedicine, IEEE Transactions on15.2 (2011): 221232.

[3] Tsai, Chia-Ling, et al. "The edge-driven dual-bootstrap iterative closest point algorithm for registration of multimodal fluorescein angiogram sequence."Medical Imaging, IEEE Transactions on 29.3 (2010): 636-649.

[4] Christian Wachinger and Nassir Navab, "Manifold learning for multimodal image registration", IEEE 13th International Conference on Modeling and Simulation, pp. 331-336, 2011.

[5] Tommy W.H Tang and Albert C.S. Chung, "Non Rigid Image Registration Using Graph Cuts",MICCAI LNCS, vol 479,pp.916-924,2007.

[6] Ronald W.K So and Albert C.S Chung, "Non Rigid Image Registration by using graph cuts with mutual information", IEEE $17^{\text {th }}$ International Conference on Image Processing, September 26-29,2010.

[7] W.H Tsai and K.S.Fu, “" Error correcting isomorphism of attributed relational graphs for pattern analysis", IEEE Trans.on SMC,vol.9,n0.12.December 1979.

[8] A.Sanifeliu and K.S.Fu,"A distance measure between attributed relational graphs for pattern recognition", IEEE Trans.on SMC,vol.13,no.3.May/June 1983.

[9] Hlaoui, Adel, and Shengrui Wang. "A Node-MappingBased Algorithm for Graph Matching." submitted (and revised) to J. Discrete Algorithms (2004).

[10] Schellewald, Christian, and Christoph Schnörr. "Subgraph matching with semidefinite programming." Electronic Notes in Discrete Mathematics 12 (2003): 279-289.

[11] Ms. Hema P Menon, and K. A. Narayanankutty. "Applicability of Non-Rigid Medical Image Registration using Moving Least Squares." International Journal of Computer Applications ,Vol.6,pp.85-92,2010.

[12] Shi, Jianbo, and Jitendra Malik. "Normalized cuts and image segmentation." IEEE Transactions on Pattern Analysis and Machine Intelligence, pp. 888-905,2000. 